\title{
$\bullet \cdot$ IJCRR \\ Section: Healthcare \\ Awareness Among College Students on the Changes in Hematological Parameters Due to COVID-19
} Sci. Journal Impact Factor: 6.1 (2018) ICV: 90.90 (2018)

\author{
Muralidharan V. A. ${ }^{1}$, Gayatri Devi R. ${ }^{2}$, Gheena S. ${ }^{3}$
}

'Saveetha Dental College and Hospitals, Saveetha Institute of Medical and Technical Sciences (SIMATS), Saveetha University, Chennai 77, India; 2Assistant Professor, Department of Physiology, Saveetha Dental College and Hospitals, Saveetha Institute of Medical and Technical Sciences (SIMATS), Saveetha University, Chennai 77, India; ${ }^{3}$ Associate Professor, Department of Oral Pathology, Saveetha Dental College and Hospitals, Saveetha Institute of Medical and Technical Sciences (SIMATS), Saveetha University, Chennai 77, India.

\section{ABSTRACT}

Introduction: COVID-19 pandemic is caused by the coronavirus which belongs to the family of retroviruses virus. It was first identified in the Wuhan province of China. The origin of the virus is unknown so as the treatment for it, as there is no vaccine found till today. Most of the people who are infected with the COVID-19 virus will experience mild to moderate respiratory illness and recover without requiring special treatment. Older people and people with medical problems like cardiovascular disease, diabetes, chronic respiratory disease, and cancer are more likely to develop a serious illness which may lead to the death of the individual. Till today there is no vaccine discovered hematological parameters are the constituents present in blood that constitutes the composition of blood such as red (RBC) and white blood cell (WBC) counts and hemoglobin (Hb) concentration are tightly regulated traits with high clinical relevance. Values outside normal ranges are diagnostic for disorders, including cancer, immune diseases, and cardiovascular disease. The COVID virus also affects these hematological parameters in a significant way. The only preventive way is social isolation.

Aim: The main of the survey is to create awareness among college students on the various changes in hematological parameters during COVID-19.

Materials and Methods: A questionnaire was prepared and administered to a hundred participants through Google forms an online platform. These study participants included college students of Saveetha dental college.

Result and Discussion: The overall result was positive as most of the study population were aware of the change in the hematological parameters due to COVID-19. This proves an outright positive result.

Conclusion: The study was conducted to analyze the awareness of the hematological changes caused due to COVID-19 virus among the college students.

Key Words: Awareness, Hematological parameters, RBC count, WBC count, Hemoglobin, COVID-19

\section{INTRODUCTION}

Blood is the most important substance that circulates throughout the body which helps in the exchange of gases, transport metabolic waste products supplying nutrients, and also acts as a defense system. It consists of many substances ${ }^{1}$ shortly known as the hematological parameters that help in maintaining the equilibrium also acting as a barrier. The current most pandemic disease that we are facing is the novel Coronavirus ${ }^{2}$. It is scientifically termed as the COVID -19 . It was first isolated in the Wuhan province in China. ${ }^{3}$ There are various opinions on the origin of the Coronavirus. It is a larger group of viruses that may cause illness in animals or humans. In humans, several Coronavirus are known ranging from the common cold to more severe diseases such as Middle East Respiratory Syndrome [MERS] and Severe Acute Respiratory System [SARS]. This COVID-19 virus belongs to the same group of viruses that were recently discovered ${ }^{1}$. Now COVID-19 is a pandemic affecting many countries globally. This COVID virus usually targets the respiratory system. Today nearly 216 countries have been affected by this. Many people are getting affected day by day. Also, the death rate is getting increased as the day passes by and it also

\section{Corresponding Author:}

R. Gayatri Devi, Department of Physiology, Saveetha Dental College and Hospitals, Saveetha Institute of Medical and Technical Sciences (SIMATS), Saveetha University, Velappanchavadi, Chennai-600077, Tamil Nadu, India; Phone: 8248016505; Email: gayatridevi@saveetha.com

ISSN: 2231-2196 (Print)

Received: 22.07 .2020
ISSN: 0975-5241 (Online)

Revised: 25.08 .2020
Accepted: 21.09 .2020 
gets spread only through the touch of contaminated goods from infected persons till day no vaccine has been found. The only way to prevent the spread of infection is through the implication of social isolation. Social isolation is a technique of state of complete lack of physical contact of an individual to another individual, more precisely even to the society. It first gets attached to the receptors of alveoli and aggregate into masses and accumulates in the alveoli of the lungs. ${ }^{4}$ The most common symptoms are fever, dry cough, and tiredness. The less common symptoms are aches and pains, sore throat, diarrhoea, conjunctivitis, headache, loss of taste or smell, and rashes or discoloration of the skin. The adverse condition's symptoms are difficulty in breathing's chest pain and loss of speech and movement. Recent studies reveal that on the entry of Coronavirus there are various changes in the hematological parameters such as RBC's, WBC's, platelets, eosinophils, etc. If the patients affected with Coronavirus have often medical complications such as anemic, hypertensive, heart disease, the patients related to diseases of blood are severely affected and the treatment given to them is also of greater difficulty. Previously our team had conducted numerous clinical trials ${ }^{5-11}$ and lab animal studies ${ }^{12-16}$ and in vitro studies ${ }^{17-19}$ and reviews on upcoming topics. The idea for this survey stemmed from the current interest in our community. Hence, the main aim of the survey is to create awareness on how the hematological parameters change due to this COVID infection among college students.

\section{MATERIALS AND METHODS}

An online survey was conducted with a self-prepared questionnaire with a sample size of hundred participants comprising students of Saveetha dental college. The questionnaire consists of questions that help in socio-economic data and questions that help in provoking awareness among the participants and questions related to facts. The participants were given a short introduction about the need to study the hematological parameters and the changes caused due to COVID-19 infection. The questionnaire was validated in a standard manner. Measures such as the selection of participants randomly, steps to prevent asking irrelevant questions to the participants placing restrictions over participant groups, and age groups are taken to minimize the bias occurring in sampling. The questionnaire was circulated using an online platform via "GOOGLE FORMS" (link:https://docs.google. com/forms/d/e/1FAIpQLSc1 15sB47zPBV0x_w5M0zZYRw7fQ-dvx4joh5LtDUqOtKbmvA/viewform?usp=sf_link).

\section{Statistical Analysis}

Descriptive Analysis was carried out using the statistical software SPSS Software version 20. The results of the survey were represented in the form of pie charts.

\section{RESULTS AND DISCUSSION}

In the current study, around $74.39 \%$ of the population responded that corona infection affects the hematological parameters and $13.41 \%$ disagreed and the rest are unaware of the hematological parameters (Figure 1). Around $41.46 \%$ responded that the patients will have a normal body temperature, $23.17 \%$ responded that the body temperature will be about 103 degree Celsius, $8.54 \%$ responded that the body temperature will be at 100 degree Celsius and $26.83 \%$ responded that it may vary from 100 degree to 103 degree Celsius (Figure 2). $74.39 \%$ of the participants were aware that there would be an increase in the body temperature on the onset of COVID-19 infection and $25.61 \%$ were unaware of it (Figure 3). $60.98 \%$ of the participants agreed that LDH level will increase on the onset of COVID infection, $21.95 \%$ of participants disagreed and $17.07 \%$ of the participants were not aware of this (Figure 4). $60.98 \%$ of the participants responded positively that due to the COVID-19 virus there will be a significant decrease in hemoglobin content in blood, $23.17 \%$ of the participants disagreed and $15.95 \%$ of the participants were not aware of this (Figure 5). $63.41 \%$ of the participants responded that due to infection in the blood by the COVID virus fever is caused, $23.17 \%$ of the participants disagreed and $13.41 \%$ of participants were not aware of this (Figure 6). $48.78 \%$ disagreed that due to COVID infection RBC will increase, $26.83 \%$ agreed that RBC cells will increase and $24.39 \%$ are not aware (Figure 7). 28.05\% strongly agreed that COVID-19 can cause anemia, $18.29 \%$ disagreed with this statement, $18.29 \%$ agreed with this, $15.85 \%$ strongly disagreed and were not aware of this fact (Figure 8). $23.2 \%$ of the participants disagreed that WBC will increase on the onset of COVID-19, 54.86\% of the participants agreed, and $21.95 \%$ of the participants were not aware of this statement (Figure 9). $15.85 \%$ of the participants disagreed that COVID-19 can lead to lymphopenia, $65.85 \%$ of the participants agreed to this and $18.3 \%$ were not aware of this (Figure 10). The correlation was done between gender and awareness of corona effects; it shows that female participants are more aware of the effects of corona than males (Figure 11). In fig 12 the bar graph shows the correlation of gender with an awareness of hemoglobin count, the responses reported that both males and females are equally aware of hemoglobin count. The correlation was done between gender with awareness of an increase in WBC count, it shows that female participants are more aware than males (Figure 13). The correlation was done between gender with awareness on increase in platelet count due to COVID-19 infection, the responses showed that female participants are more aware than males (Figure 14). The correlation was done between gender with awareness on anemia, the responses revealed that more female participants are aware of anemia than male participants (Figure 15).

The Coronavirus is a pandemic, global effects felt both physically, socially, and economically ${ }^{20}$. Recent studies conclude 
that the corona viral infection not only targets and affects the respiratory system but also has a greater influence on the blood by changing the various hematological parameters such as the RBC count, WBC count, platelet count, LDH, etc ${ }^{21}$. Many of them are not aware of this fact. The main aim of the survey is to create awareness of the changes in these hemoglobin parameters. The result is collected and the data is analyzed. It is clear that the majority of the participants are aware of it. The respondents responded properly on the effect of Coronavirus on blood ${ }^{4} .59 .4 \%$ of the participants know about the effect of Coronavirus on the hematological parameters were similar to the study of Big Wen et al. $24.6 \%$ of the participants do not agree that due to COVID infection fever is caused, as they are not aware of the symptoms of COVID infection. But the study of Yang et al showed that $98 \%$ of the participants are aware of this fact ${ }^{22}$. Nearly $65 \%$ of the participants agree that RBC, WBC, platelets will decrease. ${ }^{23}$ This is in accordance with the result analyzed by Castro et al or WHOSE survey analysis reveals that $63 \%$ of the study population are aware of this fact 24,25 . Nearly $82 \%$ of the participants found this survey useful as many of them are aware of the changes in hematological parameters. This study is conducted in a narrow range of the same homogeneous population of the students from Saveetha Dental College. In future, research work comprises a larger population to analyze the impact of COVID-19 infection on blood and its hematological parameters.

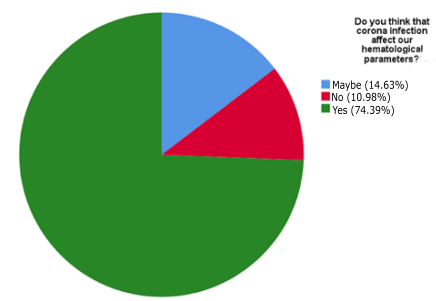

Figure 1: Pie Chart representing the percentage distribution of awareness about COVID -19 virus and changes in hematological parameters. The majority of participants $74.39 \%$ responded, "Yes" (green), 10.98\% responded, "No" (red), and 14.63\% responded, "Maybe" (blue).

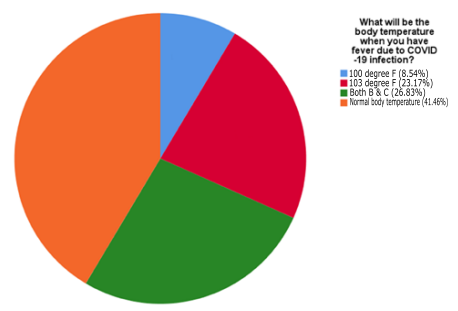

Figure 2: Pie Chart representing the percentage distribution of awareness about body temperature when you have a fever due to COVID. $8.54 \%$ responded 100 fahrenheit (blue), $23.17 \%$ responded 103 fahrenheit (red), $26.83 \%$ answered both $B$ and $C$ (green), and $41.46 \%$ answered normal body temperature (orange).

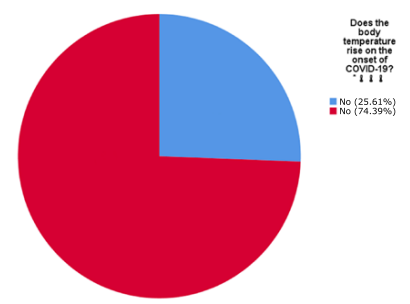

Figure 3: Pie Chart representing the percentage distribution of awareness about the body temperature rises on the onset of COVID. Majority of participants $74.39 \%$ answered, "Yes" (red) and $25.61 \%$ answered, "No" (blue).

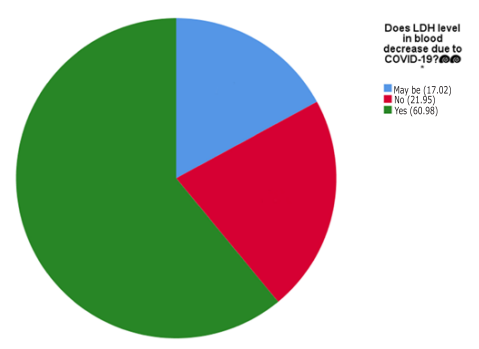

Figure 4: Pie Chart representing the percentage distribution of awareness about LDH level in blood decrease due to COVID $60.98 \%$ answered, "Yes"(green), 21.95\% answered, "No"(red), and $17.07 \%$ answered, "Maybe" (blue).

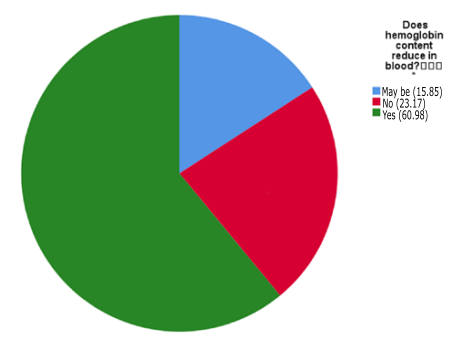

Figure 5: Pie Chart representing the percentage distribution of awareness about the hemoglobin content reduction in blood. The majority of participants $60.96 \%$ answered, "Yes" (green), 23.17\% answered, "No" (red), and 15.85\% answered, "Maybe"(blue).

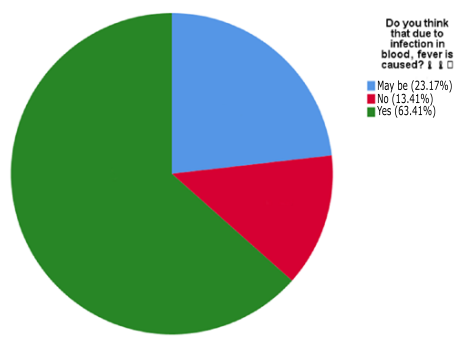

Figure 6: Pie Chart representing the percentage distribution of awareness about the infection of the blood caused fever. The majority of participants $63.41 \%$ answered, "Yes" (green), $13.41 \%$ answered, "No" (red), and $23.17 \%$ answered, "Maybe" (blue). 


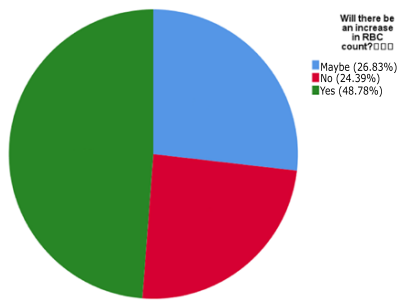

Figure 7: Pie Chart representing the percentage distribution of awareness about the increase in RBC count. The majority of participants $48.78 \%$ answered, "Yes" (green), $24.39 \%$ answered, "No" (red), and 26.83\% answered, "Maybe" (blue).

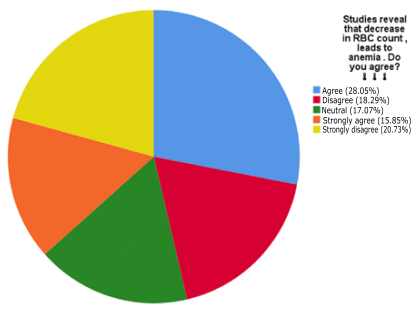

Figure 8: Pie Chart representing the percentage distribution of awareness about the decrease in RBC count leads to anemia. The majority of participants $28.05 \%$ answered agree (blue), $18.29 \%$ answered disagree (red), $17.07 \%$ answered neutral (green), 15.85\% answered strongly agreed (orange) and $20.73 \%$ answered strongly disagree (yellow).

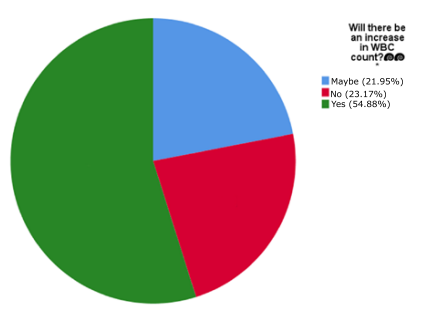

Figure 9: Pie Chart representing the percentage distribution of awareness about the increase in WBC count. The majority of participants $54.88 \%$ answered, "Yes" (green), $23.17 \%$ answered, "No" (red), and 21.95\% answered, "Maybe" (blue).

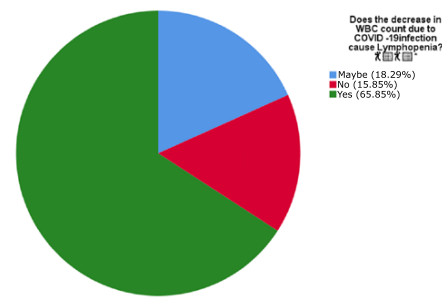

Figure 10: Pie Chart representing the percentage distribution of awareness about the decrease in WBC counts due to COVID-19 infection cause lymphopenia. The majority of participants $65.85 \%$ answered, "Yes" (green), 15.85\% answered, "No" (red), and 18.29\% answered, "Maybe" (blue).

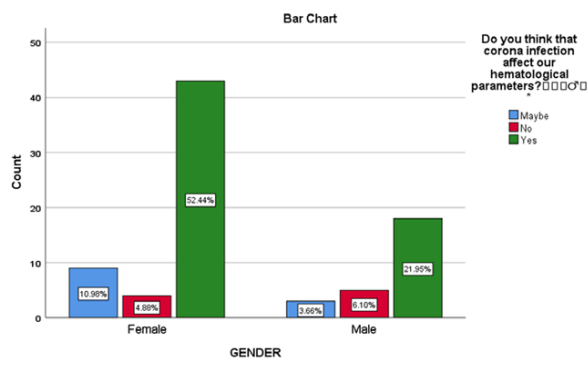

Figure 11: Bar chart showing the association between gender and the awareness of the corona effect on hematological count. X-axis represents Gender, Y-axis represents the number of individuals who are aware (green), unaware (red), and may be (blue). Out of $74.39 \%$ of participants who are aware, $21.95 \%$ constitute males and $52.44 \%$ constitute females. Females are more aware that the corona affects the hematological count than males. Pearson's Chi-square analysis $=2.749$, $P$-value $=0.253(>0.05)$ and it is not significant.

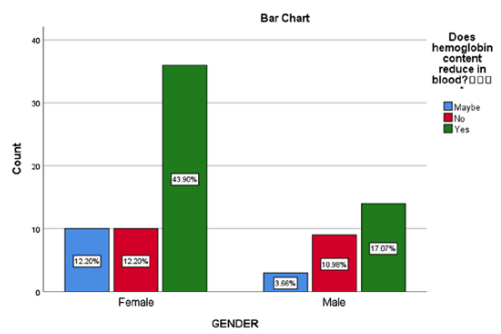

Figure 12: Bar chart showing the association between gender and the hemoglobin content reduces in blood. X-axis represents Gender, Y-axis represents individuals who are aware (green), unaware (red), and may be (blue). Out of $60.97 \%$ of participants who are aware, $43.90 \%$ constitute females and $17.07 \%$ constitute males. Females were aware of the hemoglobin content reduced in blood than males. Pearson's Chi-square analysis $=2.917$, P-value $=0.233(>0.05)$ and it is not significant.

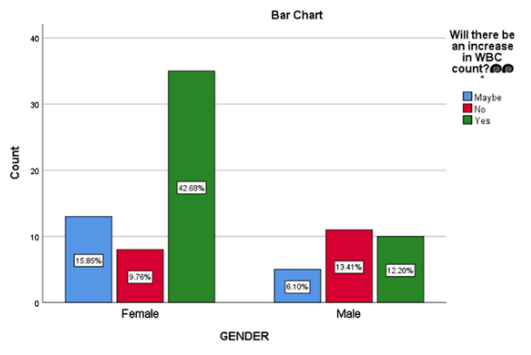

Figure 13: Bar chart showing the association between gender and increase of WBC count. X-axis represents Gender, Y-axis represents individuals who are aware (green), unaware (red), and may be (blue). Out of $54.88 \%$ of participants who are aware, $42.68 \%$ constitute females and $12.20 \%$ constitute males. More female participants were aware of the condition than male participants. Pearson's Chi-square analysis $=8.015$, $P$-value $=0.018(<0.05)$ and it is significant. 


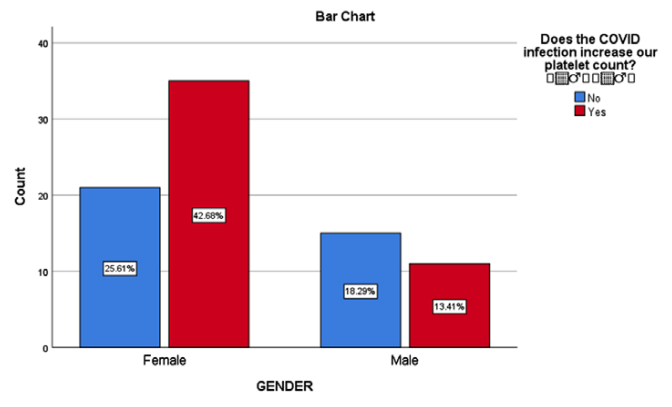

Figure 14: Bar chart showing the association between gender and COVID-19 infection increases the platelet count. X-axis represents Gender, Y-axis represents individuals. Who are aware (red) and unaware (blue). Out of $62.09 \%$ of participants who are aware, $42.68 \%$ constitute females and $13.41 \%$ constitute males. More of the female participants were aware of the condition than the male participants. Pearson's Chi-square analysis $=2.940, \mathrm{P}$-value $=0.086(>0.05)$ and it is not significant.

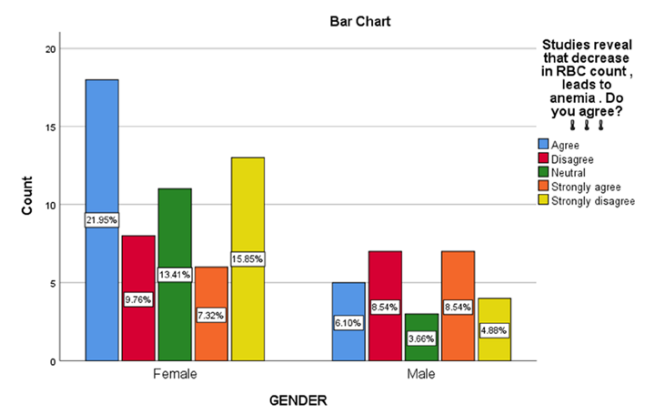

Figure 15: Bar chart showing the association between gender and study reveals that a decrease in RBC count leads to anemia. X-axis represents Gender, Y-axis represents individuals. Answered agree (blue), answered disagree (red), answered neutral (green), answered strongly agreed (orange), and answered strongly disagree (yellow). Out of $27 \%$ of participants, $21 \%$ constitute females and $6 \%$ constitute males. Females were more aware than males. Pearson's Chi-square analysis $=6.756$, P-value $=0.149(>0.05)$ and it is not significant.

\section{CONCLUSION}

From the survey, it is evident that the majority of the population are aware of the effects of COVID-19 on the hematological parameters. Since, Coronavirus has various effects on the physical and mental health, particularly the blood and its hematological parameters. As the person has a low immune system there are higher possibilities of secondary infections which may affect the health of the patient to a greater extent. Hence, a widespread awareness of COVID-19 should be done in order to achieve better health and lifestyle.

\section{ACKNOWLEDGEMENT}

We acknowledge the help offered by the institute for usage of facilities.

\section{Conflict of Interest}

All the authors declare no conflict of interest in the study

\section{Financial Support}

None

\section{REFERENCES}

1. Zhelev Z, Mollova D, Boyadziev P. Morphological and hematological parameters of Carassius Gibelio ( Pisces: Gyprinidae ) in conditions of anthropogenic pollution in Southern Bulgaria. Use of hematological parameters as biomarkers [Internet]. Trakia Journal of Science. 2016; 14: 1-15. Available from: http:// dx.doi.org/10.15547/tjs.2016.01.001

2. Al-Tawfiq JA, Hinedi K, Abbasi S, Babiker M, Sunji A, Eltigani M. Hematologic, hepatic, and renal function changes in hospitalized patients with Middle East respiratory syndrome coronavirus. Int J Lab Hematol. 2017;39(3):272-8.

3. Pise S. The Preparedness of India to Fight with COVID 19. Int J Cur Res Rev. Vol 12 Issue 06, March, 2020, 0-1, http://dx.doi. org/10.31782/IJCRR.2020.12064

4. Yarali N, Abelen YA, Unal Y, Parlakay AO. Hematological parameters and peripheral blood morphologic abnormalities in children with COVID-19. 2020; ;e28596. Available from: http:// dx.doi.org/10.22541/au.159069193.34265275

5. Samuel AR, Devi MG. Geographical distribution and occurrence of Endemic Goitre. Research Journal of Pharmacy and Technology. 2015; 8: 973- 75. Available from: http://dx.doi. org/10.5958/0974-360x.2015.00162.6

6. Baheerati MM, Gayatri Devi R. Obesity in relation to Infertility [Internet]. Research Journal of Pharmacy and Technology. 2018; 11: 3183 -85. Available from: http://dx.doi.org/10.5958/0974360x.2018.00585.1

7. Fathima F, Preetha P. Evaluation of Thyroid Function Test in Obese Patients [Internet]. Asian Journal of Pharmaceutical and Clinical Research. 2016; 9: 353 -355. Available from: http:// dx.doi.org/10.22159/ajpcr.2016.v9s3.12959

8. $\mathrm{Rj}$ I, R GD. Role of environmental factors on sleep patterns of different age groups [Internet]. Vol. 9, Asian Journal of Pharmaceutical and Clinical Research. 2016; 9: 124 - 127. Available from: http://dx.doi.org/10.22159/ajpcr.2016.v9i6.13832

9. Harsha L, Priya J, Shah KK, Reshmi B. Systemic Approach to Management of Neonatal Jaundice and Prevention of Kernicterus [Internet]. Research Journal of Pharmacy and Technology. 2015; 8: 1087 - 89. Available from: http://dx.doi.org/10.5958/0974360x.2015.00189.4

10. Dave PH, Preetha. Pathogenesis and Novel Drug for Treatment of Asthma-A Review [Internet]. Research Journal of Pharmacy and Technology. 2016; 9: 1519 - 22. Available from: http:// dx.doi.org/10.5958/0974-360x.2016.00297.3

11. Abigail, Priya J, Devi G. Evaluation of Muscular Endurance among Dentists [Internet]. Indian Journal of Public Health Research \& Development. 2019; 10: 258 -60. Available from: http://dx.doi.org/10.5958/0976-5506.2019.02808.0

12. David, Jothi Priya A, Devi G. Physical Fitness among the Dental Physician, Dental Undergraduates and Postgraduates Students [Internet]. Indian Journal of Public Health Research \& Devel- 
opment. 2019; 10: 223 - 226. Available from: http://dx.doi. org/10.5958/0976-5506.2019.02801.8

13. Shruthi M, Preetha S. Effect of Simple Tongue Exercises in Habitual Snorers [Internet]. Research Journal of Pharmacy and Technology. 2018; 11: 3614 - 18. Available from: http://dx.doi. org/10.5958/0974-360x.2018.00665.0

14. Choudhari S, Jothipriya MA. Non-alcoholic fatty liver disease [Internet]. Research Journal of Pharmacy and Technology. 2016; 9:1782 - 1785. Available from: http://dx.doi.org/10.5958/0974360x.2016.00360.7

15. Iyer PK, Gayatri Devi R, Jothi Priya A. A Survey Study on Causes, Treatment and Prevention of Onychocryptosis [Internet]. Indian Journal of Public Health Research \& Development. 2019; 10 (8): 807 -810. Available from: http://dx.doi. org/10.5958/0976-5506.2019.01990.9

16. R GD, Sethu G. EVALUATION OF ADENOIDS BY ORONASAL AND NASAL SPIROMETRY [Internet]. Asian Journal of Pharmaceutical and Clinical Research. 2018; 11(10): 272 - 275. Available from: http://dx.doi.org/10.22159/ajpcr.2018. v11i10.27365

17. Swathy S, Gowri Sethu V. Acupuncture and lower back pain [Internet]. Research Journal of Pharmacy and Technology. 2015; 8: 991 - 997. Available from: http://dx.doi.org/10.5958/0974360x.2015.00165.1

18. Renuka S, Sethu G. Regeneration after Myocardial Infarction [Internet]. Research Journal of Pharmacy and Technology. 2015; 8: 738 - 742. Available from: http://dx.doi.org/10.5958/0974360x.2015.00117.1
19. Timothy CN, Gayatri Devi R, Jothi Priya A. Evaluation of Peak Expiratory Flow Rate (PEFR) in Pet Owners [Internet]. Indian Journal of Public Health Research \& Development. 2019; $10 \mathrm{sp}(1)$ : 803 - 806. Available from: http://dx.doi. org/10.5958/0976-5506.2019.01989.2

20. Rosen RJ. Thrombotic complications in critically ill patients with COVID-19 [Internet]. Thrombosis Research. 2020; 191: 56-60. Available from: http://dx.doi.org/10.1016/j.thromres.2020.04.032

21. Elavarasan RM, Pugazhendhi R. Restructured society and environment: A review on potential technological strategies to control the COVID-19 pandemic [Internet]. Science of The Total Environment. 2020; 725: 138858. Available from: http://dx.doi. org/10.1016/j.scitotenv.2020.138858

22. Akhtar DS. Hematological changes in malaria: A comparative study [Internet]. IOSR Journal of Pharmacy and Biological Sciences. 2012; 2(4): 15-9. Available from: http://dx.doi. org/10.9790/3008-0241519

23. Lu G, Wang J. Dynamic changes in routine blood parameters of a severe COVID-19 case [Internet]. Clinica Chimica Acta. 2020; 508: 98-102. Available from: http://dx.doi.org/10.1016/j. cca.2020.04.034

24. Yavelov IS, Drapkina OM. COVID-19: hemostatic parameters and specifics of antithrombotic treatment [Internet]. Cardiovascular Therapy and Prevention. 2019; 19(3): 2571. Available from: http://dx.doi.org/10.15829/1728-8800-2020-2571

25. William A. M. The Impact of COVID-19 on Residents of Skilled Care Facilities Throughout the United States. Int J Cur Res Rev. Vol 12 Issue 16, August, 2020, 1-1, http://dx.doi.org/10.31782/ IJCRR.2020.121617

\section{QUESTIONNAIRE}

1. Do you think that corona infection affects our hematological parameters?

2. What will be the body temperature when you have fever due to COVID -19 infection?

3. Does the body temperature rise on the onset of COVID-19?

4. Does LDH level in blood decrease due to COVID-19?

5. Does hemoglobin content reduce in blood?

6. Do you think that due to infection in blood, fever is caused?

7. Will there be an increase in $\mathrm{RBC}$ count?

8. Studies reveal that decrease in $\mathrm{RBC}$ count leads to anemia . Do you agree?

9. Will there be an increase in WBC count?

10. Does the decrease in WBC count due to COVID -19infection cause Lymphopenia?

11. Will the antibodies present in our body fight against COVID-19?

12. Does the COVID infection increase our platelet count?

13. How to prevent COVID -19 spread?

14. What is the reason for the rise in temperature due to COVID -19 infection? 